\title{
On the adjustment of combined GPS/levelling/geoid networks
}

\author{
C. Kotsakis, M. G. Sideris \\ Department of Geomatics Engineering, University of Calgary, 2500 University Drive N.W., Calgary, Alberta, Canada T2N 1N4 \\ e-mail: ckotsaki@ucalgary.ca; Tel.: +1 403 2204113; Fax: +1 4032841980
}

Received: 9 September 1998 / Accepted: 8 June 1999

\begin{abstract}
A detailed treatment of adjustment problems in combined global positioning system (GPS)/levelling/ geoid networks is given. The two main types of 'unknowns' in this kind of multi-data 1D networks are usually the gravimetric geoid accuracy and a 2D spatial field that describes all the datum/systematic distortions among the available height data sets. An accurate knowledge of the latter becomes especially important when we consider employing GPS techniques for levelling purposes with respect to a local vertical datum. Two modelling alternatives for the correction field are presented, namely a pure deterministic parametric model, and a hybrid deterministic and stochastic model. The concept of variance component estimation is also proposed as an important statistical tool for assessing the actual gravimetric geoid noise level and/or testing a priori determined geoid error models. Finally, conclusions are drawn and recommendations for further study are suggested.
\end{abstract}

Key words. Geoid evaluation · GPS height transformation $\cdot$ Multi-data 1D networks $\cdot$ Adjustment

\section{Introduction}

The combined use of global positioning system (GPS), levelling, and geoid height information has been a key procedure in various geodetic applications. Although these three types of height information are considerably different in terms of physical meaning, reference surface definition/realization, observational methods, accuracy, etc., they should fulfill the simple geometrical relationship (Heiskanen and Moritz 1967)

$h-H-N=0$

Correspondence to: C. Kotsakis where $h$ are ellipsoidal heights obtained from GPS observations, $H$ are orthometric heights derived from levelling methods, and $N$ are geoid heights computed from a geoid model. In practice, Eq. (1) is never satisfied due to the following: (1) random noise in the values of $h$, $H, N$; (2) datum inconsistencies and other possible systematic distortions in the three height data sets (e.g. long-wavelength systematic errors in $N$, distortions in the vertical datum due to an overconstrained adjustment of the levelling network, deviation between gravimetric geoid and reference surface of the levelling datum, etc.); (3) various geodynamic effects (post-glacial rebound, land subsidence, plate deformation near subduction zones, mean sea level rise, monument instabilities); and (4) theoretical approximations in the computation of either $H$ or $N$ (e.g. improper or non-existent terrain/ density modelling in the geoid solution, improper evaluation of Helmert's formula for orthometric heights using normal gravity values instead of actual surface gravity observations, negligence of the sea surface topography (SST) at the tide gauges, error-free assumption for the tide gauge observations, etc.).

The statistical behaviour and modelling of the misclosures of Eq. (1), computed in a network of levelled GPS benchmarks, have been the subject of many studies which are often considerably different in terms of their research objectives. The following is a non-exhaustive list of some of these objectives. The references provided are just representative and are not the only important ones.

1. Testing the performance of global spherical harmonic models for the Earth's gravity field (International Geoid Service, IGeS 1997).

2. Testing the performance of local/regional gravimetric geoid models and their associated computational techniques (Mainville et al. 1992; Sideris et al. 1992).

3. Development of intermediate corrector surfaces for optimal height transformation between geoid surface and levelling datum surface (Mainville et al. 1997; Smith and Milbert 1996).

4. Development of corrector surfaces for long-wavelength gravimetric geoid errors (De Bruijne et al. 
1997), and general gravimetric geoid refinement strategies (Jiang and Duquenne 1996).

5. Evaluation of the achievable accuracy for 'levelling by GPS' surveys (Forsberg and Madsen 1990).

6. Monitoring, testing, and/or improving (strengthening) of already-existing vertical datums (Hein 1986; Kearsley et al. 1993).

The above list can be further extended if we substitute in Eq. (1) the GPS height $h$ with altimetric observations, and the orthometric height $H$ with the SST. A study for such marine applications is included in De Bruijne et al. (1997). In view of the many different uses for such multidata $1 \mathrm{D}$ networks, the purpose of this paper is to present some general adjustment and modelling schemes that can be employed for an optimal analysis of the misclosures of Eq. (1). In particular, we are mainly interested in applications of the types (1), (2), or (3) from the previous list. In Sect. 2, a general overview is given for various adjustment schemes that have already been applied in practice. Some general modelling considerations and the initial formulation of our models are presented in Sect. 3, and the general adjustment model is fully developed in Sect. 4. Two different cases of this model are examined in detail in Sects. 5 and 6, and finally some conclusions and recommendations are given.

\section{Overview of various adjustment/modelling schemes}

A brief review of various adjustment and modelling schemes that have already been applied for the applications mentioned in the previous section will be given here. Some general aspects of adjustment problems with combined height data sets can be found in Pelzer (1986).

\subsection{Geoid evaluation}

Most of the geoid evaluation studies, based on comparisons with GPS/levelling data, make use of the following basic model:

$h_{i}-H_{i}-N_{i}=\mathbf{a}_{i}^{\mathrm{T}} \mathbf{x}+v_{i}$

where $\mathbf{x}$ is an $n \times 1$ vector of unknown parameters, $\mathbf{a}_{i}$ is an $n \times 1$ vector of known coefficients, and $v_{i}$ denotes a residual random noise term. The parametric part $\mathbf{a}_{i}^{\mathrm{T}} \mathbf{x}$ is supposed to describe all possible datum inconsistencies and other systematic effects in the data sets. In practice, for these studies, the usual four-parameter model is often used, i.e.

$\mathbf{a}_{i}^{\mathrm{T}} \mathbf{x}=x_{\mathrm{o}}+x_{1} \cos \varphi_{i} \cos \lambda_{i}+x_{2} \cos \varphi_{i} \sin \lambda_{i}+x_{3} \sin \varphi_{i}$

and rarely its five-parameter extension (see e.g. Duquenne et al. 1995).

$$
\begin{aligned}
\mathbf{a}_{i}^{\mathrm{T}} \mathbf{x}= & x_{\mathrm{O}}+x_{1} \cos \varphi_{i} \cos \lambda_{i}+x_{2} \cos \varphi_{i} \sin \lambda_{i} \\
& +x_{3} \sin \varphi_{i}+x_{4} \sin ^{2} \varphi_{i}
\end{aligned}
$$

has also been employed. Both Eqs. (3) and (4) correspond to the following datum transformation model for the geoid undulation $N$, which is described extensively in Heiskanen and Moritz (1967, Sect. 5-9):

$$
\begin{aligned}
\Delta N_{i}= & \Delta a+\Delta X_{\mathrm{o}} \cos \varphi_{i} \cos \lambda_{i}+\Delta Y_{\mathrm{o}} \cos \varphi_{i} \sin \lambda_{i} \\
& +\Delta Z_{\mathrm{o}} \sin \varphi_{i}+a \Delta f \sin ^{2} \varphi_{i}
\end{aligned}
$$

where $\Delta X_{\mathrm{o}}, \Delta Y_{\mathrm{o}}$, and $\Delta Z_{\mathrm{o}}$ are the shift parameters between two 'parallel' datums and $\Delta f, \Delta a$ are the changes in flattening and semi-major axis of the corresponding ellipsoids. In our case, the two different datums will correspond to (1) the GPS datum and (2) the datum used for the development of the global spherical harmonic model that supports the gravimetric geoid, and for the computation of the gravity anomaly data $\Delta g$.

The model of Eq. (2) is applied to all network points and a least-squares (LS) adjustment is performed to estimate the residuals $v_{i}$, which are traditionally taken as the final external indication of the geoid accuracy. The main problem under this approach is that the $v_{i}$ terms will contain a combined amount of GPS, levelling, and geoid random error that needs to be separated into its individual components for a more reliable geoid assessment. Furthermore, an optimal adjustment in a statistical sense would require the proper weighting of the residuals, which is hardly applied in practice. Finally, the use of such oversimplified parametric models as Eqs. (3) or (4), combined with improper weighting of the residuals $v_{i}$, creates important problems in terms of the 'separability' of the various random and systematic effects between the two unknown components $\mathbf{a}_{i}^{\mathrm{T}} \mathbf{x}$ and $v_{i}$.

\subsection{Corrector surface for GPS/levelling}

The development of corrector surfaces aims basically at providing GPS users with an optimal transformation model between ellipsoidal heights $h$ and orthometric heights $H$ with respect to a given levelling datum. For a general discussion regarding theoretical and practical aspects of this problem, see Featherstone (1998). Two such developments have been reported in North America, in the US by the National Geodetic Survey (NGS; Smith and Milbert 1996) and in Canada by the Geodetic Survey Division (GSD; Mainville et al. 1997). Both studies followed a similar methodology, using initially the basic model of Eq. (2) with its parametric part given by Eq. (3). The obtained adjusted values for the residuals $v_{i}$ were then spatially modelled in a grid form using an interpolation procedure. In the GSD study a minimum-curvature interpolation algorithm was used, whereas the NGS fitted an isotropic Gaussian covariance function to the statistics of the irregularly distributed values $v_{i}$ and then used simple collocation formulas for the gridding. From the combination of the gridded values for the residuals and the adjusted values for the parameters $\mathbf{x}$, a corrector surface to the gravimetric geoid was finally computed. Some comments regarding the 'drawbacks' of these modelling approaches will be given later on in the present paper. 


\subsection{Gravimetric geoid refinement}

In De Bruijne et al. (1997) a 28-parameter surface model was estimated to correct the gravimetrically derived geoid in the North Sea area for its long-wavelength errors. TOPEX altimetric data $(h)$ and gravimetric geoid heights $(N)$ were only used in the general observation equation, Eq. (2), since the SST was neglected in this study. The parametric model $\mathbf{a}_{i}^{\mathrm{T}} \mathbf{x}$ was comprised of a simple bilinear part with four parameters (one bias, two tilts, one torsion), and a more complicated part of trigonometric polynomials with 24 coefficients. For the optimal estimation of this correction model, only the external altimetric data were properly weighted, according to their precomputed standard deviations. Extensive statistical testing was also applied to validate the final adjustment results. For the refinement of land gravimetric geoid models using GPS/levelling data, Jiang and Duquenne (1996) proposed the division of the entire test area into smaller adjacent networks, in order to better model the higher frequency geoid distortions due to the insufficient local gravity data coverage and the errors in the used Digital Terrain Model (DTM).

\subsection{Vertical datum testing/refinement}

For such applications the analyzed network usually contains a combination of some, or all, of the following data: (1) relative $\Delta H$ from conventional levelling; (2) relative $\Delta h$ from local GPS surveys; (3) $N$ or $\Delta N$ from a geoid model; (4) absolute $H$ and SST values at tide gauge stations; and (5) absolute $h$ from Satellite Laser Ranging (SLR) or global GPS campaigns. The above data configuration was proposed by Kearsley et al. (1993). In their extensive study, investigating the quality of sample subsets of the Australian Height Datum (AHD), they used the following general mathematical model for known observations and unknown parameters:

$$
\begin{aligned}
& \Delta h_{i j}=h_{j}-h_{i}+v_{\Delta h_{i j}} \\
& \Delta H_{i j}=H_{j}-H_{i}+v_{\Delta H_{i j}} \\
& \Delta N_{i j}=N_{j}-N_{i}+v_{\Delta N_{i j}} \\
& h_{j}-H_{j}-N_{j}=0
\end{aligned}
$$

All available observations $\left(\Delta h_{i j}, \Delta H_{i j}, \Delta N_{i j}\right)$, along with their a priori accuracy estimates, were simultaneously adjusted, using Eq. (6d) as a geometrical constraint for the unknown parameters at each station point $j$. For the unknown parameters, additional a priori information can also be incorporated in the adjustment algorithm (Bayesian estimation), in the form of independent point measurements with their associated variances and possible co-variances (e.g. measurement of $H$ at tide gauge sites). The above methodology suggests a powerful adjustment tool that can be used for vertical datum refinement/redefinition, where both geometrical (GPS,
SLR) and physical (levelling, geoid, mean sea level) quantities are optimally combined in a unified fashion (see also Vaniček 1991). Among the critical issues existing in this approach (as well as in the previously overviewed applications) is the estimation of the a priori covariance matrices for the different data sets. Since these types of weighting measures are only used to describe the behaviour of the random errors in the measurements, some augmentation of the observation equations, Eqs. (6), by additional auxiliary parametric models, describing possible systematic/datum offsets in the available data sets, should also be considered.

\section{General modelling considerations}

In general, Eq. (1) does not hold exactly, due not only to the presence of zero-mean random errors in the height data, but also due to a number of other direct or indirect systematic effects. Since there are not usually available a priori corrections for many of these effects, they should be individually modelled and estimated during an adjustment process. In this way, the following three general equations can be written for each point $P_{i}$ in a combined GPS/levelling/geoid (GLG) network:

$$
\begin{aligned}
h_{i} & =h_{i}^{\alpha}+f_{i}^{h}+v_{i}^{h}, \quad H_{i}=H_{i}^{\alpha}+f_{i}^{H}+v_{i}^{H}, \\
N_{i} & =N_{i}^{\alpha}+f_{i}^{N}+v_{i}^{N}
\end{aligned}
$$

where $h_{i}, H_{i}$, and $N_{i}$ denote the available 'observed' values for the GPS, orthometric and geoid heights, respectively. The superscript $\alpha$ denotes true values with respect to a unified geodetic datum, such that the following equation holds:

$h_{i}^{\alpha}-H_{i}^{\alpha}-N_{i}^{\alpha}=0$

The $f_{i}$ terms correspond to all the necessary reductions that need to be applied to the original data in order to eliminate the datum inconsistencies and other systematic errors. Finally, the $v_{i}$ terms describe zero-mean random errors, for which a second-order stochastic model is available:

$$
\mathrm{E}\left\{\mathbf{v}_{h} \mathbf{v}_{h}^{\mathrm{T}}\right\}=\mathbf{C}_{h}, \quad \mathrm{E}\left\{\mathbf{v}_{H} \mathbf{v}_{H}^{\mathrm{T}}\right\}=\mathbf{C}_{H}, \quad \mathrm{E}\left\{\mathbf{v}_{N} \mathbf{v}_{N}^{\mathrm{T}}\right\}=\mathbf{C}_{N}
$$

For the orthometric heights, the covariance $(\mathrm{CV})$ matrix $\mathbf{C}_{H}$ is known from the adjustment of the levelling network. In the same way, $\mathbf{C}_{h}$ can be computed from the adjustment of the GPS surveys performed at the levelled benchmarks. In the gravimetric geoid case, the covariance matrix $\mathbf{C}_{N}$ is computed by simple error propagation from the original noisy data used in the geoid solution; for detailed formulas, see Li and Sideris (1994). For a more realistic stochastic error model, full knowledge of the CV matrices should not be assumed. This is especially true for the geoid heights where the often vaguely known noise level of the input data (geopotential model coefficients, gravity, DTM), and the always necessary stationary noise assumption when fast spectral techniques are employed for the computations, may cause $\mathbf{C}_{N}$ to deviate considerably from reality. Hence, 
we will adopt the following stochastic model for the random noise effects in the three height data sets:

$\mathrm{E}\left\{\mathbf{v}_{h} \mathbf{v}_{h}^{\mathrm{T}}\right\}=\sigma_{h}^{2} \mathbf{Q}_{h}, \quad \mathrm{E}\left\{\mathbf{v}_{H} \mathbf{v}_{H}^{\mathrm{T}}\right\}=\sigma_{H}^{2} \mathbf{Q}_{H}$,

$\mathrm{E}\left\{\mathbf{v}_{N} \mathbf{v}_{N}^{\mathrm{T}}\right\}=\sigma_{N}^{2} \mathbf{Q}_{N}$

where the cofactor matrices $\mathbf{Q}_{h}, \mathbf{Q}_{H}$, and $\mathbf{Q}_{N}$ are assumed known from the sources previously indicated, and the three variance components are treated as unknown parameters controlling the validity of the a priori random error models. One could also extend the above stochastic model a little bit more, by decomposing the covariance matrix $\mathbf{C}_{N}$ into two different $\mathrm{CV}$ matrices with associated unknown variance components, which would correspond to the two main geoid random error sources (noisy geopotential coefficients, noisy gravity anomaly data). In this paper, the set of observation Eqs. (7) and their associated stochastic model in Eq. (10) represent the basic framework upon which all the derivations in the following sections will be based.

\section{A general adjustment model}

Let us assume that, at each point $P_{i}$ of a test network with $m$ points, we have a triplet of height observations $\left(h_{i}, H_{i}, N_{i}\right)$, or equivalently one 'synthetic' observation $l_{i}=h_{i}-H_{i}-N_{i}$. By combining Eqs. (7) and (8), we obtain the following observation equation for each network point:

$l_{i}=h_{i}-H_{i}-N_{i}=\left(f_{i}^{h}-f_{i}^{H}-f_{i}^{N}\right)+\left(v_{i}^{h}-v_{i}^{H}-v_{i}^{N}\right)$

or, in a more compact form

$l_{i}=f_{i}+v_{i}^{h}-v_{i}^{H}-v_{i}^{N}$

If the main objective for using such a test network is to evaluate the gravimetric geoid accuracy, then we are naturally interested in the estimation of the $v_{i}^{N}$ terms. Since there is a stochastic model [Eq. (10)] that has been associated with these terms, the values of $v_{i}^{N}$ are supposed to reflect all the geoid random error sources that were taken into account for the computation of the $\mathrm{CV}$ matrix $\mathbf{Q}_{N}$. Furthermore, the ability to estimate the unknown parameter $\sigma_{N}^{2}$ according to some variance component estimation algorithm (see e.g. Rao 1971, 1997), provides probably the most powerful statistical tool for a reliable estimate of the actual geoid noise, and a useful means of testing all the assumptions that were incorporated in the construction of the preliminary geoid error model $\mathbf{Q}_{N}$. There is still, however, an amount of geoid error which is not included in the $v_{i}^{N}$ terms, and for which no a priori information is available in general: aliasing effects, improper (or omitted) terrain and density modelling, various biases in the coefficients of the geopotential model, etc. Such geoid errors, which do not follow a zero-mean random behaviour, will be absorbed in the $f_{i}$ correction term along with many other systematic effects in the GPS and levelling data. In the absence of any prior statistical and/or deterministic information for these error sources, filtering them out and estimating their magnitude individually is impossible.

If, on the other hand, this test network is to be used for the determination of an optimal corrector surface for future GPS/levelling applications, then the values $f_{i}$ have to be estimated and spatially modelled in the best possible way. The random noise terms $v_{i}^{h}, v_{i}^{H}, v_{i}^{N}$ should be left out of the modelling for such a correction surface. This can be easily realized by looking at the form of the basic observation equation in a future orthometric height network which will utilize GPS/geoid information, as well as the computed corrector surface from our original test network, i.e.

$h-N-c=H+v$

where the term $c$ represents the reduction effect of the computed correction surface model. A system of equations, created by taking the differences of Eq. (13) between the GPS survey points, has now to be adjusted for the optimal estimation of the orthometric height differences $\Delta H$ with respect to the local levelling datum. Correcting, prior to this adjustment, the GPS/levelling observations for their random noise effects [which is the case if the terms $v_{i}^{h}$ and $v_{i}^{N}$ from Eq. (12) are included in the modelling of the corrector surface term $c$ ] makes no sense statistically. Furthermore, if the residual values $v_{i}^{H}$ from Eq. (12) are included in the modelling of the corrector surface, then the available original observations in Eq. (13) will be 'corrected' for an error source which does not even exist in them!

Let us now return to our initial observation model of Eq. (12). The correction term $f_{i}=f\left(P_{i}\right)$ represents a $2 \mathrm{D}$ spatial field of values, and it can be further decomposed in the general form

$f_{i}=\mathbf{a}_{i}^{\mathrm{T}} \mathbf{x}+s_{i}$

where $\mathbf{a}_{i}$ is an $(n \times 1)$ vector of known coefficients, and $\mathbf{x}$ is an $(n \times 1)$ vector of unknown deterministic parameters. The term $s_{i}$ denotes some 'residual correction', the nature of which (deterministic or stochastic) is left unspecified for now. The final observation equation for each point in the test network will therefore have the following form:

$l_{i}=\mathbf{a}_{i}^{\mathrm{T}} \mathbf{x}+s_{i}+v_{i}^{h}-v_{i}^{H}-v_{i}^{N}$

and by using matrix notation in order to combine all the network points, we obtain

$\mathbf{l}=\mathbf{A} \mathbf{x}+\mathbf{s}+\mathbf{B v}$

where

$$
\begin{aligned}
\mathbf{l} & =\left[\begin{array}{lllll}
l_{1} & \cdots & l_{i} & \cdots & l_{m}
\end{array}\right]^{\mathrm{T}}, \\
\mathbf{s} & =\left[\begin{array}{lllll}
s_{1} & \cdots & s_{i} & \cdots & s_{m}
\end{array}\right]^{\mathrm{T}}, \\
\mathbf{v} & =\left[\begin{array}{lll}
\mathbf{v}_{h}^{\mathrm{T}} & \mathbf{v}_{H}^{\mathrm{T}} & \mathbf{v}_{N}^{\mathrm{T}}
\end{array}\right]^{\mathrm{T}} \\
\mathbf{v}_{\#} & =\left[\begin{array}{lllll}
v_{1}^{\#} & \cdots & v_{i}^{\#} & \cdots & v_{m}^{\#}
\end{array}\right]^{\mathrm{T}}, \quad \#: h, H, N
\end{aligned}
$$




$$
\begin{gathered}
\mathbf{l}=\mathbf{A} \mathbf{x}+\mathbf{s}+\mathbf{B v} \quad \mathrm{E}\{\mathbf{v}\}=\mathbf{0} \\
\mathrm{E}\left\{\mathbf{v v}^{\mathbf{T}}\right\}=\mathbf{C}_{\mathbf{v}}=\left[\begin{array}{ccc}
\mathbf{C}_{h} & \mathbf{0} & \mathbf{0} \\
\mathbf{0} & \mathbf{C}_{H} & \mathbf{0} \\
\mathbf{0} & \mathbf{0} & \mathbf{C}_{N}
\end{array}\right]=\left[\begin{array}{ccc}
\sigma_{h}^{2} \mathbf{Q}_{h} & \mathbf{0} & \mathbf{0} \\
\mathbf{0} & \sigma_{H}^{2} \mathbf{Q}_{H} & \mathbf{0} \\
\mathbf{0} & \mathbf{0} & \sigma_{N}^{2} \mathbf{Q}_{N}
\end{array}\right] \\
\sigma_{h}^{2}, \sigma_{H}^{2}, \sigma_{N}^{2}: \text { unknown variance components }
\end{gathered}
$$

Fig. 1. A general model for GPS/levelling/geoid network adjustment

$$
\begin{aligned}
\mathbf{A} & =\left[\begin{array}{lllll}
\mathbf{a}_{1} & \cdots & \mathbf{a}_{i} & \cdots & \mathbf{a}_{m}
\end{array}\right]^{\mathrm{T}}, \\
\mathbf{B} & =\left[\begin{array}{lll}
\mathbf{I}_{m} & -\mathbf{I}_{m} & -\mathbf{I}_{m}
\end{array}\right], \quad \mathbf{I}_{m}: m \times m \text { unit matrix }
\end{aligned}
$$

This final adjustment model is summarized in Fig. 1. The associated stochastic model follows from the one introduced in Eq. (10).

Such adjustment problems where, apart from the unknown deterministic parameters $\mathbf{x}$ and the zero-mean random errors $\mathbf{v}$, there appear also some quantities $\mathbf{s}$ that depend on an underlying unknown function (the corrector surface in our case), are very common in geodetic applications. When the emphasis is placed on the estimation of the functionals $\mathbf{s}$, it is traditionally called a LS collocation problem with unknown parameters (Moritz 1980). In the case where the main interest is on the parameters $\mathbf{x}$, it is viewed as a simple LS adjustment problem 'in the presence of signals' (Dermanis 1978, 1984). Both approaches are of course equivalent, with an immediate relation to the classic mixed linear models of statistical theory (see e.g. Koch 1987).

The crucial point for the solution of the adjustment model in Fig. 1 is how to treat the signals $\mathbf{s}$. In a first simple deterministic approach these signals can be treated just as additional discrete unknown parameters, and their implicit relation with the underlying unknown function is completely ignored (see e.g. Dermanis 1984). This approach, however, is not applicable to our specific case of Eq. (16), because the resulting matrix of the normal equations, under the minimization principle

$\mathbf{v}^{\mathrm{T}} \mathbf{P} \mathbf{v}=\mathbf{v}_{h}^{\mathrm{T}} \mathbf{Q}_{h}^{-1} \mathbf{v}_{h}+\mathbf{v}_{H}^{\mathrm{T}} \mathbf{Q}_{H}^{-1} \mathbf{v}_{H}+\mathbf{v}_{N}^{\mathrm{T}} \mathbf{Q}_{N}^{-1} \mathbf{v}_{N}=\min$

with the weight matrix being

$\mathbf{P}=\left[\begin{array}{ccc}\mathbf{Q}_{h}^{-1} & \mathbf{0} & \mathbf{0} \\ \mathbf{0} & \mathbf{Q}_{H}^{-1} & \mathbf{0} \\ \mathbf{0} & \mathbf{0} & \mathbf{Q}_{N}^{-1}\end{array}\right]$

will always be singular. In order to obtain a unique solution, therefore, some additional constraints need to be imposed on the residual systematic corrections $s_{i}$. Two different cases will now be identified for applying these necessary constraints.

\section{A purely deterministic approach}

One easy way to solve the general adjustment model in Fig. 1 is to neglect the presence of the residual correction signals s. Essentially, this means that the corrector surface will be exclusively modelled by a preselected deterministic parametric form. In order to avoid any rank deficiency problems, the total number of selected parameters should be always smaller than the number of network points. In this case, the adjustment model of Fig. 1 will be reduced to the form

$\mathbf{l}=\mathbf{A} \mathbf{x}+\mathbf{B v}$

where $\mathbf{A}$ is some appropriate design matrix with full column rank. The final solution of Eq. (20), under the minimization principle of Eq. (18), will be given by the equations

$$
\begin{aligned}
\mathbf{W}= & \mathbf{I}_{m}-\mathbf{A}\left(\mathbf{A}^{\mathrm{T}}\left(\mathbf{Q}_{h}+\mathbf{Q}_{H}+\mathbf{Q}_{N}\right)^{-1} \mathbf{A}\right)^{-1} \\
& \times \mathbf{A}^{\mathrm{T}}\left(\mathbf{Q}_{h}+\mathbf{Q}_{H}+\mathbf{Q}_{N}\right)^{-1} \\
\hat{\mathbf{x}}= & {\left[\mathbf{A}^{\mathrm{T}}\left(\mathbf{Q}_{h}+\mathbf{Q}_{H}+\mathbf{Q}_{N}\right)^{-1} \mathbf{A}\right]^{-1} } \\
& \times \mathbf{A}^{\mathrm{T}}\left(\mathbf{Q}_{h}+\mathbf{Q}_{H}+\mathbf{Q}_{N}\right)^{-1} \mathbf{l} \\
\hat{\mathbf{v}}_{h}= & \mathbf{Q}_{h}\left(\mathbf{Q}_{h}+\mathbf{Q}_{H}+\mathbf{Q}_{N}\right)^{-1} \mathbf{W l} \\
\hat{\mathbf{v}}_{H}= & -\mathbf{Q}_{H}\left(\mathbf{Q}_{h}+\mathbf{Q}_{H}+\mathbf{Q}_{N}\right)^{-1} \mathbf{W l} \\
\hat{\mathbf{v}}_{N}= & -\mathbf{Q}_{N}\left(\mathbf{Q}_{h}+\mathbf{Q}_{H}+\mathbf{Q}_{N}\right)^{-1} \mathbf{W l} \\
\hat{\mathbf{v}}_{\text {total }}= & \mathbf{B} \hat{\mathbf{v}}=\hat{\mathbf{v}}_{h}-\hat{\mathbf{v}}_{H}-\hat{\mathbf{v}}_{N}=\mathbf{W l}
\end{aligned}
$$

In the case where a full CV matrix for the height data noise is not available, but only some gross estimates for the mean height accuracy are known, a much simpler version of the above equations occurs. If we denote by $q_{h}^{2}, q_{H}^{2}$, and $q_{N}^{2}$ the a priori uniform accuracy estimates for the ellipsoidal, orthometric, and geoid heights, respectively, then we obtain the following solution:

$\mathbf{W}=\mathbf{I}_{m}-\mathbf{A}\left(\mathbf{A}^{\mathrm{T}} \mathbf{A}\right)^{-1} \mathbf{A}^{\mathrm{T}}$

$\hat{\mathbf{x}}=\left(\mathbf{A}^{\mathrm{T}} \mathbf{A}\right)^{-1} \mathbf{A}^{\mathrm{T}} \mathbf{l}$

$\hat{\mathbf{v}}_{h}=\frac{q_{h}^{2}}{q_{h}^{2}+q_{H}^{2}+q_{N}^{2}} \mathbf{W} \mathbf{l}$

$\hat{\mathbf{v}}_{H}=-\frac{q_{H}^{2}}{q_{h}^{2}+q_{H}^{2}+q_{N}^{2}} \mathbf{W} \mathbf{l}$

$\hat{\mathbf{v}}_{N}=-\frac{q_{N}^{2}}{q_{h}^{2}+q_{H}^{2}+q_{N}^{2}} \mathbf{W} \mathbf{l}$

From the last three equations [also from Eqs. (21c)(21e)] the crucial role of the stochastic model for the random noise in the height data is obvious. It offers the means of applying an optimal filtering to the total residuals $\mathbf{B} \hat{\mathbf{v}}=\mathbf{W} \mathbf{l}$ of the adjustment by separating the noise coming from each individual height component. It is rather interesting, though a highly unrealistic case, 
that when stationary white noise has been assumed for all height data types, the estimates for the unknown parameters $\hat{\mathbf{x}}$ and the total residuals $\mathbf{B} \hat{\mathbf{v}}$ will not depend at all on the three different noise levels $q_{h}^{2}, q_{H}^{2}$, and $q_{N}^{2}$. By applying covariance propagation to the above results, the $\mathbf{C V}$ matrix $\mathbf{C}_{\hat{\mathbf{x}}}$ of the adjusted model parameters can be also determined, which should always be used to evaluate the quality of the parametric corrector surface for future GPS/levelling applications. Another useful matrix is the cross-CV matrix between the adjusted model parameters and the adjusted residuals for the various height data sets, from which important information can be extracted regarding the correlation of the corrector surface with the available data.

The reliability of the previous results depends on (1) the suitability of the parametric model Ax to describe effectively all the systematic effects in the height data sets, and (2) the correctness of the stochastic model for the observational noise $\left(\mathbf{Q}_{h}, \mathbf{Q}_{H}, \mathbf{Q}_{N}\right)$. It is therefore necessary to estimate also the three unknown variance components (see Fig. 1). The method of variance component estimation traditionally used in geodesy is Rao's Minimum Norm Quadratic Unbiased Estimation MINQUE (Rao 1971). In the geodetic literature this problem has been solved independently, for a variety of adjustment models, by many researchers; an extensive review with further references to the relevant literature is given in Grafarend (1985). The following algorithm follows the MINQUE criterion and computes optimal estimates for the unknown variance components of the ellipsoidal heights $\left(\hat{\sigma}_{h}^{2}\right)$, orthometric heights $\left(\hat{\sigma}_{H}^{2}\right)$, and geoid heights $\left(\hat{\sigma}_{N}^{2}\right)$ :

$\hat{\boldsymbol{\sigma}}=\mathbf{J}^{-1} \mathbf{k}$

$\hat{\boldsymbol{\sigma}}=\left[\begin{array}{lll}\hat{\sigma}_{h}^{2} & \hat{\sigma}_{H}^{2} & \hat{\sigma}_{N}^{2}\end{array}\right]^{\mathrm{T}}$
$\mathbf{k}_{i}=\hat{\mathbf{v}}_{i}^{\mathrm{T}} \mathbf{Q}_{i}^{-1} \hat{\mathbf{v}}_{i} \quad i, j: h, H, N$

$$
\begin{aligned}
\mathbf{J}_{i j}=\operatorname{tr}[ & \left(\mathbf{Q}_{h}+\mathbf{Q}_{H}+\mathbf{Q}_{N}\right)^{-1} \mathbf{W} \mathbf{Q}_{i} \\
& \left.\times\left(\mathbf{Q}_{h}+\mathbf{Q}_{H}+\mathbf{Q}_{N}\right)^{-1} \mathbf{W} \mathbf{Q}_{j}\right]
\end{aligned}
$$

There are occasions, however, where the use of algorithm (23) may lead to negative estimates for the unknown variance components. In such cases, a modification of the MINQUE method is required (see e.g. Sjoberg 1984; Rao 1997). A number of statistical tests and subsequent iterations are finally needed in order to validate the adjustment results. An overview of the whole adjustment procedure described in this section is given in the flowchart of Fig. 2.

\section{A ‘collocation’ approach}

The main disadvantage of the previous adjustment approach is the difficulty of finding a good parametric model Ax to describe all the possible systematic inconsistencies in the height data sets (too many effects to model). This, in turn, causes problems with respect to how reliable the results would be for the GPS/levelling/ geoid noise residuals and for the corresponding variance components. The use of classical statistical testing may help to identify, to some degree, the weakness of a specific parametric model, but it cannot provide the means for model improvement. It should be noted that a good parametric model does not necessarily imply small values for the estimated residuals $\mathbf{B} \hat{\mathbf{v}}$, since the noise level in the original height data $\left(\mathbf{Q}_{h}, \mathbf{Q}_{H}, \mathbf{Q}_{N}\right)$ may allow relatively large values. It is the accuracy $\mathbf{C}_{\hat{\mathbf{x}}}$ of the adjusted model parameters that should determine how good a model is, and how effectively it can be further used in future GPS/levelling applications.

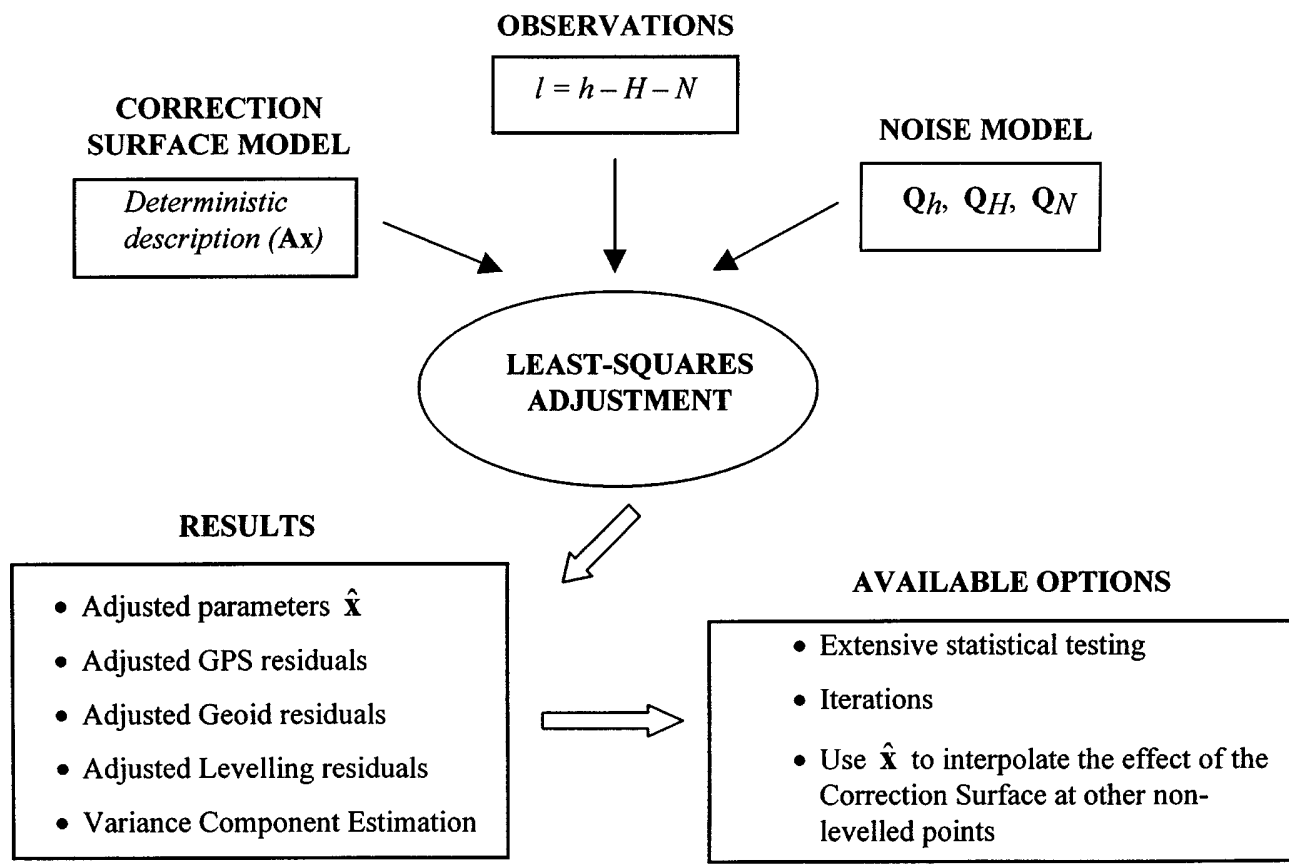

Fig. 2. Flowchart for the adjustment procedure in the deterministic approach 
Although the parameterization of the distortion effects in combined GLG networks is a very interesting topic on its own, it may be more appropriate not to try putting all the systematic errors in a preselected parametric form. For small networks, in particular, this should be the guiding rule, since in such cases only a small number of unknown deterministic parameters can be introduced in order to keep the degrees of freedom relatively large and the reliability of the adjustment results relatively high. Such a 'compact' model is of course unable to fully describe the complexity of the various systematic effects and it should be accompanied by additional residual corrections, which were previously introduced in the form of signals $\mathbf{s}$ [see Eq. (16)]. The solution of the general adjustment model in Fig. 1 requires the incorporation of the signals $\mathbf{s}$ in the minimization principle, which now takes the form

$\mathbf{s}^{\mathrm{T}} \mathbf{Q}_{s}^{-1} \mathbf{s}+\mathbf{v}_{h}^{\mathrm{T}} \mathbf{Q}_{h}^{-1} \mathbf{v}_{h}+\mathbf{v}_{H}^{\mathrm{T}} \mathbf{Q}_{H}^{-1} \mathbf{v}_{H}+\mathbf{v}_{N}^{\mathrm{T}} \mathbf{Q}_{N}^{-1} \mathbf{v}_{N}=\min$

with $\mathbf{Q}_{s}^{-1}$ being an appropriate weight matrix for the unknown correction signals.

Although the solution obtained by using Eq. (24) does not necessarily have to admit a stochastic interpretation for the signal part, it is useful to consider the signals as additional stochastic parameters, just like the zero-mean random errors $\mathbf{v}$; an excellent discussion on this aspect can be found in Dermanis (1984). The stochasticity of $\mathbf{s}$ is actually necessary in the case where statistical tests related to the validity of their weight (covariance) model $\mathbf{Q}_{s}$ are to be applied.

One of the main difficulties in this approach is that the mean value $\mathbf{m}_{s}=\mathrm{E}\{\mathbf{s}\}$ of the stochastic signals will not necessarily be zero, due to the systematic behaviour that is supposed to exist in their values. As a result, $\mathbf{m}_{s}$ should appear in the final estimation formulas if we are seeking unbiased estimators (i.e. equivalence between the LS principle of Eq. (24) and the best linear unbiased estimation (BLUE) for $\mathrm{E}\{\mathbf{s}\} \neq \mathbf{0}$; for detailed formulas see e.g. Dermanis 1987). In order to avoid such computationally useless estimates, we can initially solve the system $\mathbf{I}=\mathbf{A x}+\mathbf{s}+\mathbf{B v}$ using Eq. (24) with a unit signal weight matrix. The initial solution for the signal part,

$$
\begin{aligned}
\mathbf{W}= & \mathbf{I}_{m}-\mathbf{A}\left(\mathbf{A}^{\mathrm{T}}\left(\mathbf{Q}_{h}+\mathbf{Q}_{H}+\mathbf{Q}_{N}+\mathbf{I}_{m}\right)^{-1} \mathbf{A}\right)^{-1} \\
& \times \mathbf{A}^{\mathrm{T}}\left(\mathbf{Q}_{h}+\mathbf{Q}_{H}+\mathbf{Q}_{N}+\mathbf{I}_{m}\right)^{-1} \\
\hat{\mathbf{s}}_{\text {init }}= & \left(\mathbf{Q}_{h}+\mathbf{Q}_{H}+\mathbf{Q}_{N}+\mathbf{I}_{m}\right)^{-1} \mathbf{W} \mathbf{l}
\end{aligned}
$$

can be viewed as the 'smoothest' residual correction field that best fits the available observations $\mathbf{l}$, the selected parametric model $\mathbf{A x}$, and the associated stochastic model for the random noise effects $\left(\mathbf{Q}_{h}, \mathbf{Q}_{H}, \mathbf{Q}_{N}\right)$.

Now, we can easily compute the overall trend in the signals $\mathbf{s}$ by fitting some smooth surface to the $\hat{\mathbf{s}}_{\text {init }}$ values. If we evaluate this fitted surface at the test network points, we obtain in general some values $\hat{\mathbf{m}}_{\mathbf{s}} \neq \hat{\mathbf{s}}_{\text {init }}$. We can then create the following 'reduced' observations and signals: $\mathbf{l}_{r}=\mathbf{l}-\hat{\mathbf{m}}_{\mathbf{s}}$

$\mathbf{s}_{r}=\mathbf{s}-\hat{\mathbf{m}}_{\mathbf{s}}$

It is now safe to assume that the reduced signals $\mathbf{s}_{r}$ have zero mean, i.e. $\mathrm{E}\left\{\mathbf{s}_{r}\right\}=\mathbf{0}$. Furthermore, the numerical values $\left(\hat{\mathbf{s}}_{\text {init }}-\hat{\mathbf{m}}_{\mathbf{s}}\right)$ can be used for an empirical determination of a covariance function model describing the average spatial behaviour of the reduced signals $\mathbf{s}_{r}$. In this way, we can repeat the adjustment of the model in Fig. 1, using a new 'improved' version for the stochastic model of the correction signals:

$\mathbf{l}_{r}=\mathbf{A} \mathbf{x}+\mathbf{s}_{r}+\mathbf{B} \mathbf{v}$

$\mathrm{E}\left\{\mathbf{s}_{r}\right\}=\mathbf{0}, \quad \mathrm{E}\left\{\mathbf{s}_{r} \mathbf{s}_{r}^{\mathrm{T}}\right\}=\mathbf{C}_{s_{r}}=\sigma_{s_{r}}^{2} \mathbf{Q}_{s_{r}}$

The elements of the cofactor matrix $\mathbf{Q}_{s_{r}}$ are computed from the empirical CV model estimated at the previous step. An unknown variance component has been also introduced in order to diagnose any problems related to the validity of the empirical signal covariance function. The solution of the adjustment model in Eq. (27) will be given by the following unbiased estimators:

$$
\begin{aligned}
\mathbf{W}= & \mathbf{I}_{m}-\mathbf{A}\left(\mathbf{A}^{\mathrm{T}}\left(\mathbf{Q}_{h}+\mathbf{Q}_{H}+\mathbf{Q}_{N}+\mathbf{Q}_{s_{r}}\right)^{-1} \mathbf{A}\right)^{-1} \\
& \times \mathbf{A}^{\mathrm{T}}\left(\mathbf{Q}_{h}+\mathbf{Q}_{H}+\mathbf{Q}_{N}+\mathbf{Q}_{s_{r}}\right)^{-1} \\
\hat{\mathbf{x}}= & {\left[\mathbf{A}^{\mathrm{T}}\left(\mathbf{Q}_{h}+\mathbf{Q}_{H}+\mathbf{Q}_{N}+\mathbf{Q}_{s_{r}}\right)^{-1} \mathbf{A}\right]^{-1} } \\
& \times \mathbf{A}^{\mathrm{T}}\left(\mathbf{Q}_{h}+\mathbf{Q}_{H}+\mathbf{Q}_{N}+\mathbf{Q}_{s_{r}}\right)^{-1} \mathbf{l}_{r} \\
\hat{\mathbf{v}}_{h}= & \mathbf{Q}_{h}\left(\mathbf{Q}_{h}+\mathbf{Q}_{H}+\mathbf{Q}_{N}+\mathbf{Q}_{s_{r}}\right)^{-1} \mathbf{W} \mathbf{l}_{r} \\
\hat{\mathbf{v}}_{H}= & -\mathbf{Q}_{H}\left(\mathbf{Q}_{h}+\mathbf{Q}_{H}+\mathbf{Q}_{N}+\mathbf{Q}_{s_{r}}\right)^{-1} \mathbf{W} \mathbf{l}_{r} \\
\hat{\mathbf{v}}_{N}= & -\mathbf{Q}_{N}\left(\mathbf{Q}_{h}+\mathbf{Q}_{H}+\mathbf{Q}_{N}+\mathbf{Q}_{s_{r}}\right)^{-1} \mathbf{W} \mathbf{l}_{r} \\
\hat{\mathbf{s}}_{r}= & \mathbf{Q}_{s}\left(\mathbf{Q}_{h}+\mathbf{Q}_{H}+\mathbf{Q}_{N}+\mathbf{Q}_{s_{r}}\right)^{-1} \mathbf{W l}_{r}
\end{aligned}
$$

The final solution equations are similar to the ones obtained under the deterministic approach, with the only differences being (1) the use of reduced observations $\mathbf{I}_{r}$ instead of the original $\mathbf{l}$, and (2) the incorporation of the signal covariance matrix $\mathbf{Q}_{s_{r}}$. In the special case of stationary white noise in all three height data sets, no significant simplification of the above formulas occurs due to the appearance of the matrix $\mathbf{Q}_{s_{r}}$. The estimation of the four unknown variance components $\hat{\sigma}_{h}^{2}, \hat{\sigma}_{H}^{2}, \hat{\sigma}_{N}^{2}, \hat{\sigma}_{s .}^{2}$ follows a straightforward extension of the MINQUE algorithm of Eq. (23) and it is omitted.

As it was mentioned in Sect. 5, various statistical tests and iterative solutions can be performed in order to finally validate the adjustment results. For statistical testing procedures in extended adjustment models with signals, see Dermanis and Rossikopoulos (1991) and the references given therein. In any case, a complete answer 
for the estimated corrector surface should include: (1) the estimated parametric model $\mathbf{A} \hat{\mathbf{x}}$, (2) the parameters describing the non-zero mean signal trend ( $\hat{\mathbf{m}}_{s}$ are just the values of this trend at the test network points); (3) the estimated values for the reduced zero-mean signals $\hat{\mathbf{s}}_{r}$ at the network points; and (4) a covariance model for the zero-mean signals $\mathbf{s}_{r}$. A combined use of (3) and (4), in a collocation-type prediction formula, is required for the interpolation of the zero-mean part of the correction signal at other non-levelled points. A general flowchart for the whole computational procedure described in this section is given in Fig. 3a, b.

\section{Summary and conclusions}

The use of combined GPS/levelling/geoid networks definitely provides a very attractive evaluation scheme for the accuracy of gravimetric geoid models. At the same time, GLG networks constitute the skeleton of 'common points' in the attempt to find optimal transformation models between GPS and orthometric heights. These are two different problems which, never- theless, can be attacked simultaneously through a unified adjustment setting. As far as the geoid evaluation problem is concerned, a GLG network adjustment can essentially be used for testing the reliability of preliminary geoid error models, which have been derived via internal error propagation from the source data and their noise used in the gravimetric solution. Variance component estimation has been proposed as a useful statistical tool for computing and testing the actual geoid noise level. The important role played by the stochastic noise model of the other two height components was also demonstrated through the derived filtering equations for the total noise residuals of the adjustment. This general approach also allows us to check individually various additive geoid error models (see comment at the end of Sect. 3). In the absence of any prior geoid error model, we can still use a unit weight matrix and obtain an estimate for the a posteriori unit weight variance for the geoid noise.

For the problem of modelling a corrector surface to the gravimetric geoid for GPS-to-orthometric height transformation, it is important to filter out all the zeromean random noise effects coming from the triplet of the

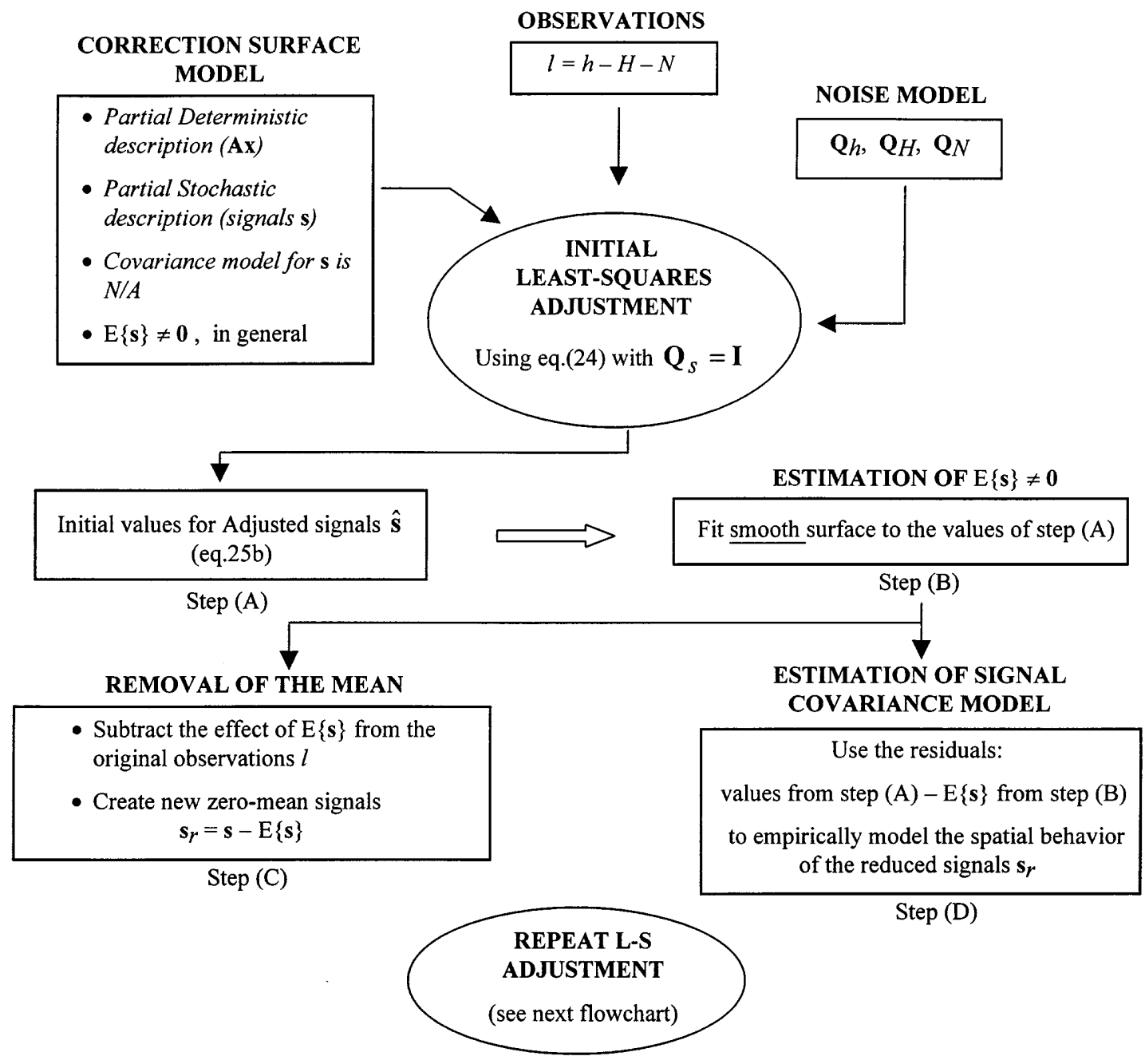

Fig. 3. a Flowchart for the adjustment procedure in the 'collocation' approach. b Flowchart for the adjustment procedure in the 'collocation' approach (continued) 


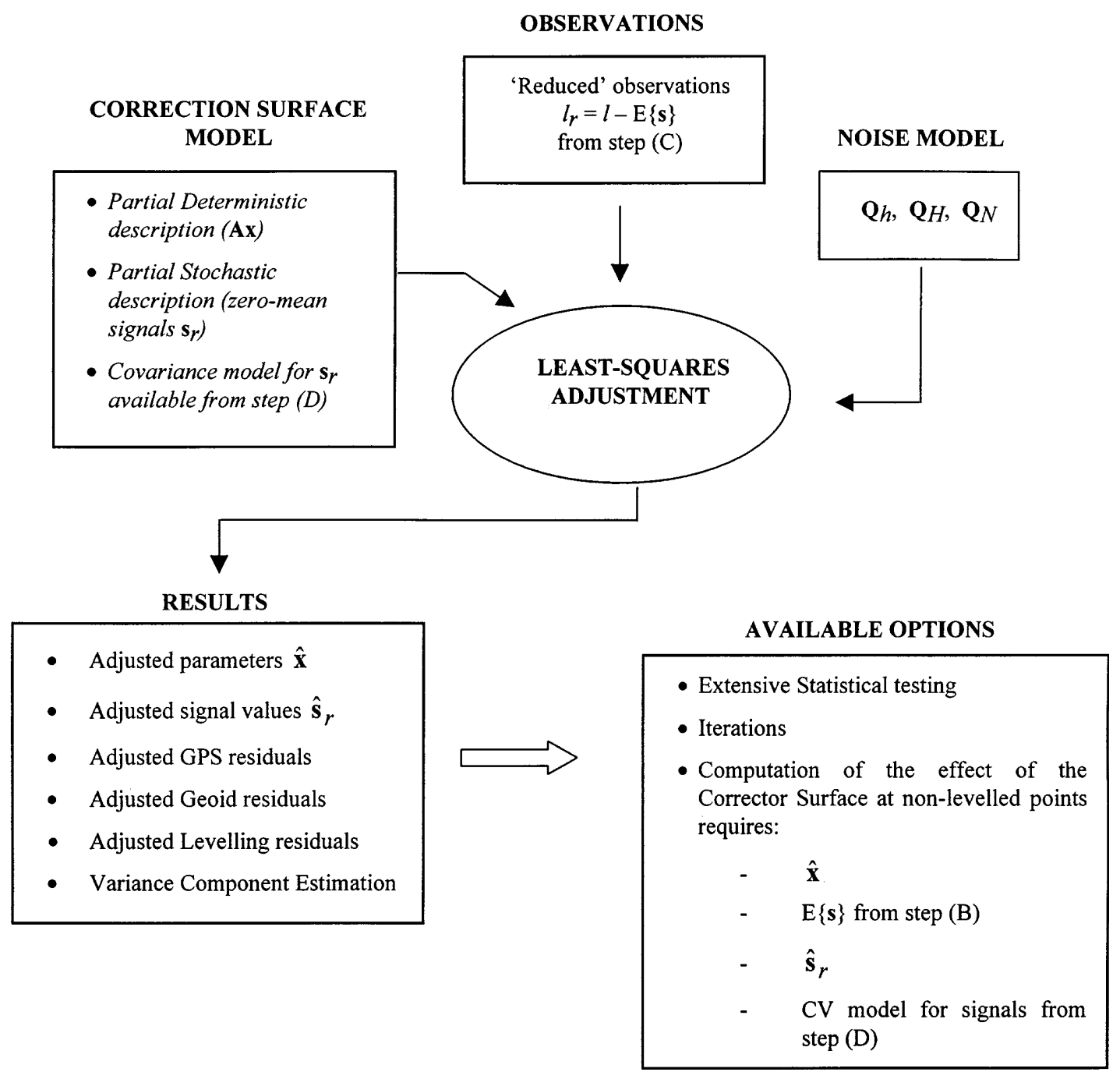

Fig. 3b

height data. This is the main weakness of some of the presently available attempts for such a modelling. This correction surface will also absorb a part of the geoid long-wavelength error which does not necessarily follow a zero-mean random behaviour, and which is not generally possible to be explicitly isolated. Two modelling alternatives have been presented for the description of the systematic correction field. These are (1) purely discrete deterministic modelling and (2) hybrid deterministic and 'stochastic' modelling. Again, the tool of variance component estimation provides the statistical means to test the admissibility of the correction field's $\mathrm{CV}$ model when (2) is employed.

The problem of statistical testing for various hypotheses, regarding the a priori accuracy information and the modelling choices in GLG networks needs to be addressed in more detail, especially in view of the many different levels of accuracy desired by GPS/levelling users. In this direction, the problem of optimization and design of GLG networks is another important and interesting topic that certainly needs to be explored. Although the present paper was restricted to a theoretical framework only, numerical work using the proposed methodologies is well underway and it will be presented in a future paper.

Acknowledgements. The authors would like to thank Prof. Dr. Erik Grafarend, who kindly presented the material of this paper at the IV Hotine-Marussi Symposium on Mathematical Geodesy, Trento, Italy, 14-17 September 1998. Financial support was provided through a contract with the Geodetic Survey Division of Geomatics Canada.

\section{References}

De Bruijne AJT, Haagmans RHN, de Min EJ (1997) A preliminary North Sea Geoid Model GEONZ97. Project report, Directoraat-Generaal Rijkswaterstaat, Meetkundige Dienst

Dermanis A (1978) Adjustment of geodetic observations in the presence of signals. Int School of Advanced Geodesy, 2nd course: 'Space-time geodesy, differential geodesy, and geodesy in the large', Lecture notes, Erice, Italy, 18 May -2 June

Dermanis A (1984) Signals in geodetic networks. Int School of Advanced Geodesy, 3nd course: 'Design and optimization of geodetic networks', Lecture notes, Erice, Italy, 25 April-10 May

Dermanis A (1987) Geodetic applications of interpolation and prediction. Int School of Geodesy A. Marussi, 4th course: 
'Applied and basic geodesy: present and future trends', Lecture notes, Erice, Italy, 15-25 June

Dermanis A, Rossikopoulos D (1991) Statistical inference in integrated geodesy. Paper presented at IUGG XXth General Assembly, Vienna, 11-21 August

Duquenne H, Jiang Z, Lemarie C (1995) Geoid determination and levelling by GPS: some experiments on a test network. IAG Symposia Gravity and Geoid, No 113, pp 559-568, SpringerVerlag

Featherstone W (1998) Do we need a gravimetric geoid or a model of the Australian height datum to transform GPS heights in Australia? Austr Surv 43(4): 273-280

Forsberg R, Madsen F (1990) High-precision geoid heights for GPS levelling. Proc 2nd Int Symp Precise Positioning with the Global Positioning System, Ottawa 3-7 September, pp 1060-1074

Grafarend E (1985) Variance-Covariance estimation, theoretical results and geodetic applications. Statist Decisions, Suppl Iss 2: 407-441

Hein GW (1986) Height determination and monitoring with time using GPS observations and gravity data. In: Pelzer $\mathrm{H}$, Niemeier W (eds) Determination of heights and height changes. Contributions to the Symposium on Height Determination and Recent Vertical Crustal Movements in Western Europe, Hannover, 15-19 September pp 349-360

Heiskanen WA, Moritz H (1967) Physical geodesy. WH Freeman, San Francisco

International Geoid Service (1997) The Earth gravity model EGM96: testing procedures at IGeS. Sp Iss, Bull n.6, DIIAR, Politechnico di Milano, Italy

Jiang Z, Duquenne H (1996) On the combined adjustment of gravimetrically determined geoid and GPS levelling stations. J Geod 70: 505-514

Kearsley AHW, Ahmad Z, Chan A (1993) National height datums, levelling, GPS heights and geoids. Aust J Geod Photogram Surv 59: $53-88$

Koch K-R (1987) Parameter estimation and hypothesis testing in linear models. Springer, Berlin Heidelberg New York
Li YC, Sideris MG (1994) Minimization and estimation of geoid undulation errors. Bull Geod 68: 201-219

Mainville A, Forsberg R, Sideris MG (1992) Global positioning system testing of geoids computed from geopotential models and local gravity data: a case study. J Geophy Res 97 (B7): $11,137-11,147$

Mainville A, Craymer M, Blackie S (1997) The GPS height transformation 1997, an ellipsoidal-orthometric height transformation for use with GPS in Canada. Report of Geodetic Survey Division, Geomatics Canada, Ottawa

Moritz H (1980) Advanced physical geodesy. Herbert Wichmann, Karlsruhe

Pelzer H (1986) Height determination - adjustment models for combined data sets. In: Pelzer H, Niemeier W (eds) Determination of heights and height changes. Contributions to the Symposium on Height Determination and Recent Vertical Crustal Movements in Western Europe, Hannover, 15-19 September pp 327-340

Rao CR (1971) Estimation of variance components - MINQUE theory. J Multivar Statist 1: 257-275

Rao PSRS (1997) Variance components estimation: mixed models, methodologies, and applications. Monographs on statistics and applied probability, vol 78. Chapman \& Hall, London

Sideris MG, Mainville A, Forsberg R (1992) Geoid testing using GPS and levelling (or GPS testing using levelling and the geoid?). Aust J Geod Photogram Surv 57: 62-77

Sjoberg L (1984) Non-negative variance component estimation in the Gauss-Helmert adjustment model. Manuscr Geod 9: 247280

Smith DA, Milbert DG (1996) The GEOID96 high resolution geoid height model for the United States. National Oceanic and Atmospheric Administration (NOAA), US National Geodetic Survey, Silver Springs, MD

Vaniček P (1991) Vertical datum and NAVD88. Surv Land Inf Syst 51(2): 83-86 